\title{
Contrasting B cell- and T cell-based protective vaccines
}

\author{
Vincent A.A. Jansen ${ }^{\mathrm{a}, *}$, Hester Korthals Altes ${ }^{\mathrm{b}}$, Georg A. Funk ${ }^{\mathrm{a}}$, Dominik Wodarz ${ }^{\mathrm{c}}$ \\ ${ }^{a}$ School of Biological Sciences, Royal Holloway-University of London, Egham, Surrey TW20 OEX, UK \\ ${ }^{\mathrm{b}}$ Population Biology, Institute for Biodiversity and Ecosystem Dynamics, University of Amsterdam, P.O.Box 94084, 1090 GB Amsterdam, \\ The Netherlands \\ ${ }^{\mathrm{c}}$ Department of Ecology and Evolution, University of California, 321 Steinhaus Hall, Irvine, CA 92697, USA
}

Received 17 May 2004; received in revised form 2 November 2004; accepted 3 November 2004

Available online 13 December 2004

\begin{abstract}
A substantial research effort is devoted to the development of vaccines based on T cells. Such a vaccine would provide a means to protect against infection with HIV and stop the current pandemic. Here we investigate the possibility to develop a protective T cellbased vaccine. We do this by means of a mathematical model which describes the dynamics of a pathogen and the immune system in the early stages of infection. We compare an immune response that is near immediate - as is the case for a humoral response-with that of a response in which the effector cells have to be formed from precursor cells - as occurs in T cell responses. The latter applies to a $\mathrm{T}$ cell-based vaccine. A near immediate response is associated with a threshold number of effector cells above which an infection cannot take hold. For a T cell-based vaccine this threshold increases with the amount of antigen the immune system is exposed to. For small initial doses, as one would naturally expect to occur, this gives rise to impractically large thresholds. Thus, although a $\mathrm{T}$ cell vaccine might work against a high dose exposure, it might fail when exposed against to a low-dose exposure. This limits, we argue, the efficacy of $\mathrm{T}$ cell-based vaccines.
\end{abstract}

(C) 2004 Elsevier Ltd. All rights reserved.

Keywords: Vaccine; Mathematical model; Human immunodefiency virus

\section{Introduction}

The processes which occur during an infection are highly complex as they involve a multitude of actors and interactions. Verbal reasoning is often insufficient to predict the response of the immune system to the pathogen quantitatively. Mathematical models allow us to go beyond verbal reasoning and can help to understand and quantify the dynamics of the immune system and predict the outcomes of interactions and interventions. Here we will formulate and analyse a basic model for the within-host dynamics of the immune system. Our model describes the immune system's reaction to an antigen. It has been inspired by the model for the

\footnotetext{
*Corresponding author. Tel.: + 441784443179 ; fax: + 441784470756

E-mail address: vincent.jansen@rhul.ac.uk (V.A.A. Jansen).
}

dynamics of viruses and the immune system as developed by Nowak and co-workers (see e.g. Nowak and May, 2000; Perelson, 2000).

Traditionally, vaccines have been based on antibody mediated protection from infection, and hinge on longlasting memory generated through B cells combined with an immediate response of antibodies to antigen upon challenge. This approach has so far been unsuccessful in generating protective vaccines against most persistent infections, in particular against the human immunodefiency virus (HIV). Therefore the possibility of vaccines based on $\mathrm{T}$ cell responses has received much attention. While memory $\mathrm{T}$ cells can be maintained at high levels following immunization, this does not necessarily correlate with protection (see Johnson, 2002; Maecker and Maino, 2003 for reviews). Unfortunately, no successful protective vaccine based on $\mathrm{T}$ cells has yet been produced. To investigate the 
extent to which it is theoretically possible to produce a protective vaccine based on $\mathrm{T}$ cell responses we study two models and contrast a cellular and humoral immune response in the early phases of infection.

An effective vaccine requires memory. The precise mechanism underlying immunological memory is not known. However, the effect of memory, i.e. elevated levels of certain immune cells for some time after stimulation, is textbook knowledge. Here, we model memory by letting some class of immune cells decay slower than other classes following stimulation. For this paper we prefer this heuristic approach over a mechanistic description of memory (McLean, 1994; Antia et al., 1998; Utzny and Burroughs, 2001) because our main interest is in the consequences of memory. Because secondary antibody responses are generated almost instantaneously from very high-affinity antibodies we assume that for B cells memory is at the level of the effector cells, whereas for $\mathrm{T}$ cells a response is based on the reactivation of precursors which can be long lived and serve as memory cells. Although the details are not known, this is generally thought to be the mechanism underlying $\mathrm{T}$ cell activation from memory (Zinkernagel, 2002).

\section{Vaccines based on humoral immune responses}

Our model is a stylized description of the process of viral infection and the host's immune reaction. The main aim of this section is to provide a simple description of the humoral immune response to contrast this with a response based on T cells. We therefore gloss over much of the detail of the humoral immune response.

The basic humoral immune response model has four variables: uninfected target cells $(T)$, infected target cells $(I)$, free virus $(V)$ and the humoral immune response $(B)$. Target cells are formed with a rate $\lambda$, this rate can depend on the density of target cells but for reasons of ease and clarity we will not consider this possibility here. Target cells are lost at rate $\delta T$. The rate of infection is $\beta V T$, where $\beta$ is the transmission parameter. Infected cells die with rate $\gamma I$ and upon death release $c$ virus particles (alternatively one can interpret this as a death rate $\gamma I$ and a production of virus through shedding of $c \gamma I)$. Virus particles are neutralized through antibodies. We assume that the antibody concentration is proportional to the concentration of $\mathrm{B}$ cells. The rate of removal of virus is given by $k B V$. The parameter $k$ is the removal parameter. In the absence of infections or an immune response the virus concentration will decay at rate $\phi V$.

Upon contact with antigen, B cells proliferate and release antibodies at a rate proportional to the amount of free virus, $a B V$. In the absence of proliferation, the immune response decreases exponentially with rate $\mu B$.
For reasons of clarity and simplicity we model the humoral immune response with a single variable, $B$. We do this because the antibody release is fast upon contact of $\mathrm{B}$ cells with antigen, and the antibody concentration is therefore proportional to the B cell concentration. Although antibody release depends on the availability of $\mathrm{T}$ cell help, for reasons of simplicity we assume that the amount of help is not a limiting factor. We have not modelled the input of $\mathrm{B}$ cells from the bone marrow in detail, because this rate is very small in comparison with the input through proliferation. Because the aim of this model is to describe the dynamics of the virus and the immune system in early infection or immediately upon challenge we only describe virus specific B cells that can act against the antigen upon infection. We do not model the dynamics of memory cells in detail but effectively assume that memory B cells and effector cells behave the same, apart from possibly their death rates. We assume the dynamics are dominated by the most persistent cells and hence the parameter $\mu$ represents the lowest death rate of $\mathrm{B}$ cells.

The basic model for virus B cell dynamics reads:

$$
\begin{aligned}
& \frac{\mathrm{d} T}{\mathrm{~d} t}=\lambda-\beta V T-\delta T, \\
& \frac{\mathrm{d} I}{\mathrm{~d} t}=\beta V T-\gamma I, \\
& \frac{\mathrm{d} V}{\mathrm{~d} t}=c \gamma I-\phi V-k B V, \\
& \frac{\mathrm{d} B}{\mathrm{~d} t}=a B V-\mu B .
\end{aligned}
$$

In the absence of virus, the target cells are maintained at a level $\hat{T}=\lambda / \delta$. When a virus infects the host, the virus will multiply in the available target cells. Initially, the immune response is weak but as the infection develops an immune response will be mounted. As a consequence, the number of infected cells will decrease. If the per cell decay rate of B cells, $\mu$, is sufficiently low, the number of cells remains high long after the number of infected cells has decreased, thus providing immunological memory. This simple model gives a heuristic but effective description of the interaction between virus and immune system. We will use this model to study the criteria needed for protection from infection.

Vaccination involves stimulation of the immune system through administration of a suitable antigen. When stimulation levels off the humoral immune response will have increased to a certain level, denoted $B_{v}$; from that moment on the immune response will slowly start to decrease. This decay is approximately exponential and can be described by $B(t)=B_{v} \mathrm{e}^{-\mu t}$. To predict how long vaccination protects from infection we need to calculate the level of the immune response conferring protection, $B_{\text {threshold }}$. 


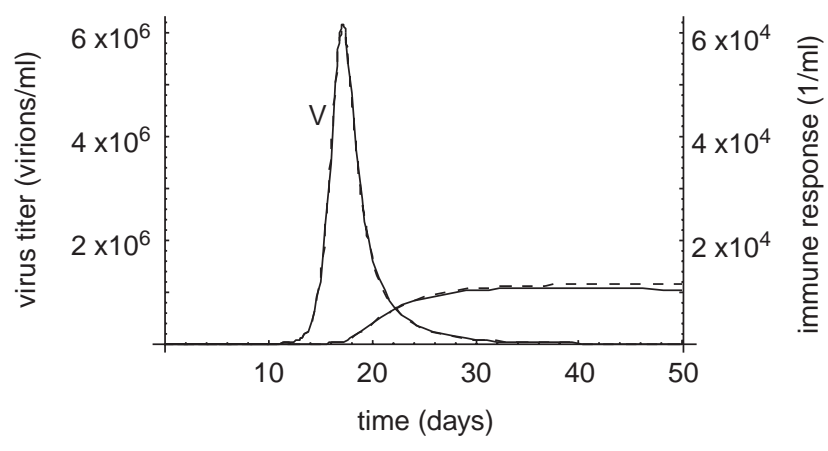

Fig. 1. The change in the humoral response during an infection. The drawn line represents a numerical solution of Eqs. (1), for the dashed line we set $\mu=0$, corresponding to the humoral response with infinite memory as given by (2). Parameters: $\lambda=1400, \mu=0.0033, \gamma=0.25$, $\delta=0.0025, \quad \beta=0.0000001, \quad \phi=32, \quad c=4000, \quad k=0.025, \quad a=$ $0.000000222, T(0)=560,000, I(0)=0, V(0)=0.04, E(0)=50$.

Fig. 1 shows the dynamics during an infection. The immune response will increase as long as antigen is present. The growth rate of the virus will initially be high but will decrease throughout infection, because of the depletion of the target cell population and the mounting immune response. Eventually, this will reduce the viral numbers to very low levels or clear the infection (see Fig. 1). During the early stages of infection the main factors affecting the immune response are the release of antigen and proliferation. The lifetime of memory B cells is, independently of antigen, at least 150 days and probably much larger (Maruyama et al., 2000; Takahashi et al., 2001). Based on this observation we assume the decay of $\mathrm{B}$ cells is negligible on the timescale of the primary infection. Taking this into account we can approximate the dynamics of the immune system during early infection as

$$
\frac{\mathrm{d} B}{\mathrm{~d} t}=a V B
$$

This provides a good description during the early stages of the infection (Fig. 1). An infection normally starts with the introduction of a relatively small number of virus particles in the host and can establish itself if this small number can increase to large numbers. This will only be the case if, on average, each virus particle gives rise to at least one new virus particle in a host with a target cell population that is not yet changed due to the presence of the virus. In epidemiology the average number of secondary infections per primary infection in a pathogen-free population is known as the basic reproductive number or $R_{0}$ (Anderson and May, 1991). In viral population dynamics, the basic reproductive number is the average number of secondary infected cells arising from a single infected cell in a host that is otherwise free of virus. If $R_{0}>1$ the amount of virus grows through a chain reaction of new infections, if $R_{0}<1$ the amount of virus (or infected cells) steadily decreases and the infection fizzles out.

The basic reproductive number $R_{0}$ is calculated as follows: each infected cell produces $c$ virus particles. The average lifetime a virus particle is $1 /(\phi+k B)$, so that the infectious potential of an infected cell is given by $\beta c /(\phi+k B)$. The number of target cells at the onset of the infection is $\hat{T}$. Hence, an infected cell at the beginning of an infection can give rise to $R_{0}=$ $\beta c \hat{T} /(\phi+k B)$ newly infected cells per unit of time. Whether or not an infection takes off depends on the level of the immune response. Protection from infection is provided if the strength of the immune response exceeds the critical level $B_{\text {threshold }}$. At this threshold, we find ourselves at the knife's edge between a successful and unsuccessful infection and hence $R_{0}=1$. This level can now be calculated from $\beta c \hat{T} /\left(\phi+k B_{\text {threshold }}\right)=1$ which is equivalent to

$B_{\text {threshold }}=\frac{\beta c \hat{T}-\phi}{k}$.

We can now work out how long a vaccine will provide protection. As long as the level of the immune response exceeds $B_{\text {threshold }}$ the infection cannot take off. Therefore, the period of time over which the vaccinated host is protected can be calculated from $B_{v} \mathrm{e}^{-\mu t}=B_{\text {threshold }}$, where $B_{v}$ is the immune response generated through vaccination, which gives

$t=\frac{1}{\mu} \ln \frac{B_{v}}{B_{\text {threshold }}}=\frac{1}{\mu} \ln \frac{k B_{v}}{\beta c \hat{T}-\phi}$.

This formula tells us that a vaccine provides long protection if the loss of immune cells is slow and the immune system has a long memory ( $\mu$ small), if the immune response against the virus is effective (high $k$ ) or if it elicits a strong response (high $B_{v}$ ) albeit that the two latter effects only work in the logarithm of the response. Long protection is also associated with a low transmission rate $\beta$, with a scarcity of target cells or with a small burst size, $c$. These factors are mostly properties of the virus, less amenable to directed human manipulation and therefore of less interest in the context of vaccine models.

What happens if the initial level of immune response is just below the threshold level? Does the viral population initially expand, then to be nipped in the bud by an immune response that increases through proliferation of the effector cell population? We numerically calculated the peak number of infected cells, which we use as a proxy for the severity of the infection, as a function of the initial level of the immune response (see Fig. 2). In agreement with the above analysis, we found that if the immune response exceeds the threshold level, $B_{\text {threshold, }}$, protection from infection can be achieved. 


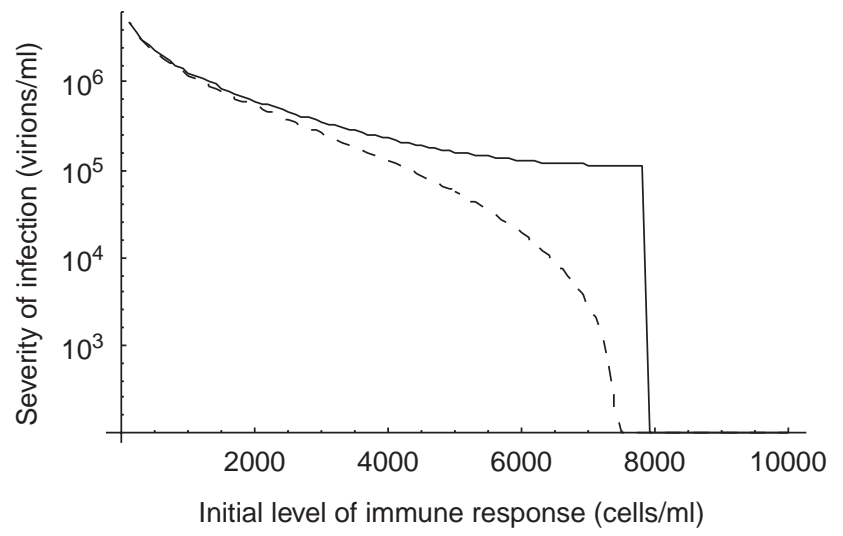

Fig. 2. The severity of the infection, measured as the peak number of infected cells, as a function of the concentration of B cells present at the time of the infection $\left(B_{v}\right)$. Two scenarios are considered: finite memory $(\mu=0.0033$, continuous line), or infinite memory $(\mu=0$, dashed line). The infection is considered to be cleared if the number of virions falls below the value in the beginning of the infection (at $t=1$ ). The infection starts with at $V(0)=100$, parameters as in Fig. 1. For these parameters $B_{\text {threshold }}=7680$.

If the immune response falls below this threshold, the severity of infection depends on the level of memory. If there is no loss of immunity following vaccination, i.e. if memory is infinite, the severity of the infection decreases with the initial level of immunity in a continuous fashion. For the more realistic case of finite memory, protection is granted as long as immunity is maintained above the critical level. If the immunity falls below the threshold the infection abruptly becomes more severe and we can see a jump in the severity of the infection. It can be shown by approximating the orbits around the equilibrium, that the size, in terms of infected cells or viremia, of this jump is approximately $2 \mu / a$. This discontinuity is caused by the fact that a loss of immunity will result in a delay between infection and an increase in the immune response. If the level of the immune response is below the threshold, this delay allows for the expansion of the virus population before an immune response comes up and controls the infection. Note that near the threshold level the growth of the viral population is slow, because the reproductive number is close to unity and that therefore this effect is noticeable, even if the waning of the immunity is slight.

A second consequence of finite memory is the possibility of a persistent infection. Our model predicts that the virus load at equilibrium is $\mu / a$ : finite memory therefore can allow for chronic infection. In the model the dynamics always go to equilibrium following introduction of the virus. Despite the fact that clearance of the virus is rare after the virus has established itself (Gruters et al., 2002) one would expect that clearance is possible if the viral load is sufficiently low. If one assumes a threshold below which the infection is cleared, clearance of infection can be achieved if the level of the immune response is sufficiently high at the onset of infection (see Fig. 2).

If an infection persists the strength of the immune response can be used as a benchmark for the efficacy of a vaccine. During a chronic infection the immune response will go to a more or less constant level which can easily be measured. It can be shown that in order to prevent infection the immune response elicited by a vaccine needs to be greater than during chronic infection (Korthals Altes et al., 2002). This can be seen as follows (Bonhoeffer et al., 2000): let the immune response during a chronic infection be given by $B_{\text {chronic }}$ and the number of target cells by $T_{\text {chronic }}$. The average number of infected cells per infected cells is $\beta c T_{\text {chronic }} /(\phi+$ $\left.k B_{\text {chronic }}\right)=1$. This is because in the chronic phase of infection, the host has stable viremia: in other words, the average number of cells infected by a single cell is one. By combining this with Eq. (3) and after elimination of $\phi$ we find: $B_{\text {threshold }}=B_{\text {chronic }}+(\beta c / k)\left(\hat{T}-T_{\text {chronic }}\right)$. Hence, the threshold level of the immune response must exceed the level found during chronic infection, if infection decreases the number of target cells. This provides a benchmark for the efficacy of vaccines against pathogens causing chronic infection. A similar benchmark for the protective threshold can be found by relating the threshold level of the immune response to the basic reproductive number, $R_{0}$, of a virus in a newly infected host. The parameter $R_{0}$ is often easier to estimate than the number of target cells (see e.g Little et al., 1999). Using the expression for the basic reproductive number, $\mathrm{R}_{0}$, given earlier in this section, we can write $R_{0}=\beta c \hat{T} / \phi$, assuming the immune response in the naive host was negligible. Substituting $c \beta \hat{T}$ in the expression for the threshold protection level we find $B_{\text {threshold }}=\left(R_{0}-1\right) \phi / k$.

There are experimental data linking the initial level of vaccine-induced immunity to protection against infection. One study indicates that in individuals who had a severe exposure to rabies virus a single booster inoculation 4 years after the initial (post-exposure) treatment was sufficient to maintain a rabies specific antibody titer that would probably confer protection against a second exposure (Fayaz et al., 1981). In another study a threshold titer (with human complement) was found that was likely to confer protection to meningococcal disease (Welsch and Granoff, 2004). In this study protection in the absence of bactericidal activity by complement was associated with significantly higher concentrations and avidities of antibodies directed against a capsular protein of the meningococcus.

\section{Vaccines based on $T$ cell responses}

In this section we will adapt our model and use it to describe vaccines based on $\mathrm{T}$ cell responses. $\mathrm{T}$ cells act 
on infected cells and therefore we do not need to consider free virus. Each infected cell gives rise to $c$ virus particles and hence the rate of infection is given by $\beta c I T$. $\mathrm{T}$ cells come in two forms: precursor cells, $P$, and effector cells, $E$. Precursor $\mathrm{T}$ cells proliferate into effector cells upon contact with antigen; we assume that the rate of activation of precursor cells increases linearly with the amount of antigen, $I$, and is given by $b P I$ (see De Boer and Perelson (1994) and Wodarz and Jansen (2001) for a detailed derivation of proliferation functions). On average, a cell that proliferates gives rise to $n$ effector cells. Stimulation leads to a programmed number of cell divisions followed by a contraction of the number of cells, after which a number of memory cells remain (Kaech and Ahmed, 2001; van Stipdonk et al., 2001; Blattman et al., 2002; Badovinac et al., 2002; Antia et al., 2003). The number of cell divisions is about $7-10$, so $n$ will be in the order of 100-200 per precursor. Precursor cells are removed at rate $\mu$. Precursor cells can also be formed directly in the thymus but this contribution is negligible compared to the number formed through proliferation. Effector cells are removed at rate $v$. Because effector cells are short lived and the rate of removal of effector cells is much higher than the removal rate of precursor cells, we take $v \gg \mu$. Effector cells remove infected cells at a rate $k E I$. Effector cells contribute to memory. The fine details of this process are unknown but it is known that after proliferation effector cells are programmed to die, with a few turning into memory cells (Jacob and Baltimore, 1999; Seder and Ahmed, 2003). We therefore assume that, in keeping with this, effector cells turn into memory cells at a constant rate, $m E$.

We assume that memory cells are long-lived cells that, upon contact with antigen, proliferate into effector cells. We further assume here that memory cells and precursors have similar average life times and proliferation rates. This can be justified by observing that in the long run the cells with the slowest death rates predominate. We therefore assume precursors and memory cells are functionally equivalent. The model reads

$\frac{\mathrm{d} T}{\mathrm{~d} t}=\lambda-\beta c I T-\delta T$,

$\frac{\mathrm{d} I}{\mathrm{~d} t}=\beta c I T-\gamma I-k E I$,

$\frac{\mathrm{d} P}{\mathrm{~d} t}=m E-b P I-\mu P$,

$\frac{\mathrm{d} E}{\mathrm{~d} t}=b n P I-v E-m E$.

A numerical solution to these equations is given in Fig. 3. For our simulations we parametrized the model on an HIV infection using the estimates parameters

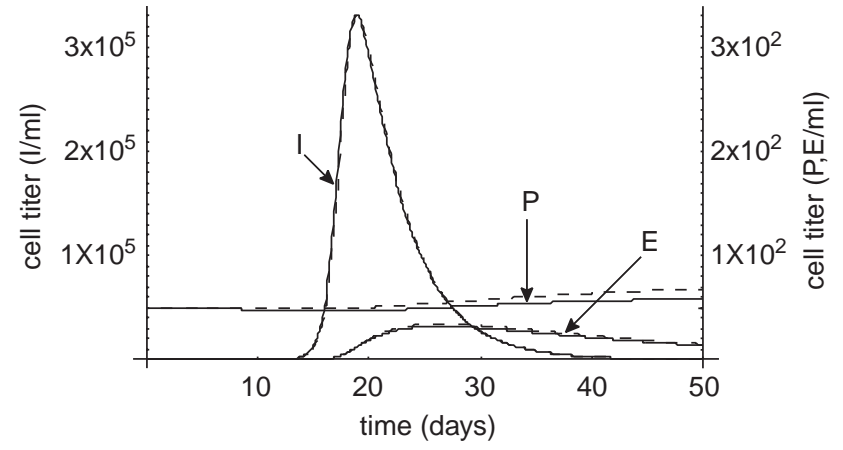

Fig. 3. The change in infected cells $(I)$ and precursor $(P)$ and effector $\mathrm{T}$ cells $(E)$ during an infection. The drawn line represents a numerical solution of Eqs. (4) $(\mu=0.0125)$, the dashed line a numerical solution to Eq. (6) $(\mu=0)$. Parameters: $\lambda=1400, \gamma=0.25, \delta=0.0025, \beta=$ $0.0000031, \quad k=0.0002, \quad \mu=0.0033, \quad m=0.023, \quad v=0.022, \quad b=$ 0.0000000044 and $n=100$. For these values $b(n m /(v+m)-1)=$ 0.000000222 , which is the value of $a$ in Fig. 1 . Initial values: $T(0)=$ $560,000, I(0)=0.00001, P(0)=50$ and $E(0)=0$. This choice of parameters was based on values for the dynamics of HIV given in Funk et al. (2001).

given in Funk et al. (2001). Comparing this figure with Fig. 1, in which a similar result for a B cell response is shown, we see that the $\mathrm{T}$ cell response results in fewer infected cells. However, the T cell response takes longer to control the infection and much longer to clear it. Note that the $\mathrm{T}$ cell response is not particularly good at responding to the virus at low levels.

We will next investigate to what extent $T$ cell vaccines can prevent infection, and repeat the calculations made in the previous section to estimate the duration of protection. In the absence of antigen and virus, there is neither proliferation nor activation and the $\mathrm{T}$ cell equations of the model simplify to

$\frac{\mathrm{d} P}{\mathrm{~d} t}=m E-\mu P$,

$\frac{\mathrm{d} E}{\mathrm{~d} t}=-(v+m) E$.

This system has eigenvalues $-\mu$ and $-v+m$. The effector population declines exponentially. If the levels just after vaccination are given by $E_{v}$ for the effector cells, the decay is described by: $E(t)=E_{v} \mathrm{e}^{-(v+m) t}$. If the number of precursor cells just after vaccination is given by $P_{v}$ the number of precursors is given by

$P(t)=P_{v} \mathrm{e}^{-\mu t}+E_{v} \frac{m}{v+m-\mu}\left(\mathrm{e}^{-\mu t}-\mathrm{e}^{-t(v+m)}\right)$.

If $v+m \gg \mu$ this will quickly converge to $P(t)=\left(P_{v}+\right.$ $\left.E_{v} m /(v+m-\mu)\right) \mathrm{e}^{-\mu t}$. We aim to predict how long a vaccine will provide protection. As in the case of $\mathrm{B}$ cells, the virus cannot increase in numbers if the effector population exceeds a threshold level, which in this case is given by $E_{\text {threshold }}=(\beta c \hat{T}-\gamma) / k$. However, because effector cells are short lived, this will not lead to 
long-lasting protection. Using the formula derived for the decay of the effector cells we find that protection last for $[1 /(v+m)] \ln E_{v} / E_{\text {threshold }}$ units of time. The factor $1 /(v+m)$ is the average lifetime of an effector cell, which is in the order of 45 days (Ogg et al., 1999). Protection based on effector $\mathrm{T}$ cells can be expected to be of similar order of magnitude. If protection depends on the availability of effector $\mathrm{T}$ cells very frequent boosting or a form of continuous stimulation is required.

By providing a stronger stimulus the duration of the protective period can be increased, but in order to triple this period a 20 -fold increase $(\ln 20 \approx 3)$ in the amount of antigen is required, assuming effectors increase linearly with antigen. To increase the protective period by a factor 10 a 22,000 -fold increase $(\ln 22,000 \approx 10)$ in the initial number of effector cells is required. The result that the threshold level of effector cells has to exceed the level found during a chronic infection and the relation to $R_{0}$ also holds for $\mathrm{T}$ cell vaccines (Korthals Altes et al., 2002).

There remains the possibility that a vaccine can be based on precursor or memory cells, which have a longer lifespan than effector cells. To gain a quantitative understanding of the proliferation of $\mathrm{T}$ cells upon infection we assume that the loss of precursors is negligible during infection. If this is the case, the $\mathrm{T}$ cells dynamics are given by

$$
\frac{\mathrm{d} P}{\mathrm{~d} t}=m E-b P I
$$

$\frac{\mathrm{d} E}{\mathrm{~d} t}=b n P I-v E-m E$.

To analyse the potential of $\mathrm{T}$ cells to stem an infection, we assume here that the dynamics of effector $\mathrm{T}$ cells are much faster than the dynamics of precursor $\mathrm{T}$ cells. Thus we can make a quasi-steady-state assumption for the equation describing the change in effector cells. Setting $\mathrm{d} E / \mathrm{d} t=0$ we get $E=b n P I /(v+m)$. This quasisteady-state assumption assumes that the lifetime of effector cells is short, i.e. $v+m$ is large, and that the number of effector cells formed is small, i.e. that $b n P I$ is small. (This analysis can be generalized by a change of variables to hold if $b n P I$ is not small (see the appendix).) Substituting this into the formula for the basic reproductive number: $R_{0}=\beta c \hat{T} /(\gamma+k E)=1$ and solving for $P$ we obtain

$P_{\text {threshold }}=\frac{\nu+m}{n b I} \frac{\beta c \hat{T}-\gamma}{k}$.

We have assumed that $v+m$ is large, in addition, in the beginning of an infection the number of infected cells, I, will be small. Therefore this threshold will be large, unless the proliferation constant, $b$, the killing constant, $k$, or the burst size, $n$, are very large. This also demonstrates that even if a challenge using a large dose might create sufficient effector cells to reduce the reproductive number below one, challenges using a smaller dose might not do so. Therefore a $\mathrm{T}$ cell vaccine that responds well to a challenge using a large dose in an experimental setting, might still prove unsuitable against small doses of inoculum because these will not activate sufficient effector cells. This is an important and somewhat counterintuitive result. These considerations make it unlikely that, under these assumptions, a vaccine based on $\mathrm{T}$ cells can be developed that protects from infection in practice.

Our argument so far has been based on the assumption that $T$ cell dynamics are fast and that the fraction of effector cells equilibrates quickly. This assumption is, however, unlikely to be justified. Using Eqs. (8) and (9) from the appendix it can be shown that the fraction $F$ equilibrates with rate

$(v+m) \sqrt{\left(1+\frac{b I}{v+m}\right)^{2}+4 \frac{b I}{v+m}\left(\frac{n m}{v+m}-1\right)}$.

If the number of infected cells is large equilibration will be fast. However, in the beginning of the infection the number of infected cells is small and the rate of equilibration is approximately $v+m$, and the characteristic return time is $1 /(v+m)$, which corresponds to the average life time of an effector CD8 T cell, which is in the order of 6 weeks. Most infections increase at a rate that is much faster, HIV for instance has a doubling time of 10-12 h (Little et al., 1999). Therefore, the assumption that change in the number of infected cells is slow compared to the $\mathrm{T}$ cell dynamics is not justified, and the effector numbers will lag behind the number of infected cells, reducing the efficacy of a $T$ cell vaccine even further.

To find out the dynamics in the beginning of the infection we performed numerical simulations. These show that the $\mathrm{T}$ cells will eventually control the infection, but that compared to Fig. 1, the infection decreases slower in the final phase than when infection is controlled by the humoral immune response (see Fig. 3). Also clearance of the infection, if it occurs at all, takes much longer: at low viral densities effector cells cannot be maintained at high levels. However, the severity of the infection, as measured by the maximum number of infected cells, tends to be smaller if the infection is controlled by $\mathrm{T}$ cells.

By increasing the number of precursors cells at the time of the infection the severity of the infection can be reduced. However, in contrast to the vaccine based on a humoral response, there is no visible threshold level of precursor cells for which the severity drops abruptly (cf. Fig. 2 with Fig. 4). For a vaccine based on T cells there is no practically relevant level of precursor cells for which protection is guaranteed if the initial amount of antigen is low (Fig. 4, there is a protective threshold but 


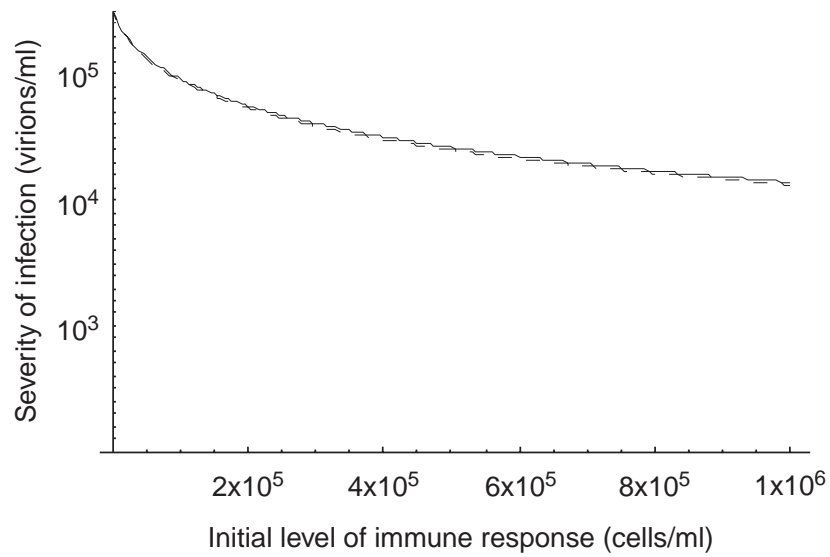

Fig. 4. The severity of the infection as a function of the concentration of precursor $\mathrm{T}$ cells present at the time of the infection. The infection is considered to be cleared if the number of infected cells falls below the value in the beginning of the infection (at $t=1$ ). The infection is inoculated at $I(0)=0.00001$. Parameters as in Fig. 3 .

only for an astronomically large number of precursor cells). This is an important observation. This happens because effector $\mathrm{T}$ cells are formed from precursors, a process which is dependent on the presence of antigen. In the $\mathrm{T}$ cell model the precursor population will give rise to a population of effector cells, but the number of cells activated depends on the amount of antigen. For small amount of antigen therefore $\mathrm{T}$ cells are not able to control an emerging infection the same way that the humoral immune response can. For this reason $\mathrm{T}$ cells are much less suitable for protective vaccines than a humoral immune response.

\section{Discussion}

Vaccines normally protect through humoral responses (Zinkernagel, 2000). There are a number of diseases against which no protective vaccines have been developed, notably malaria, leprosy, HCV and HIV. For all these diseases the $\mathrm{T}$ cell response is essential for control (Zinkernagel, 2002). Especially for HIV the need for an effective vaccine is pressing to control the current pandemic.

Experimental vaccines have been successful at eliciting strong $\mathrm{T}$ cell responses. However, so far they have at best provided partial protection against infection (Johnson, 2002). Despite major efforts no effective protective $\mathrm{T}$ cell vaccine has yet been developed. McMichael et al. (2002) postulate that the reason why $\mathrm{T}$ cell vaccines are so much more difficult to design is that antibodies can neutralize the virus before it infects a cell whereas $\mathrm{T}$ cells can only neutralize a cell once it is infected (Robbins et al., 1995). This implicitly assumes that the antibodies neutralize each and every virus particle, but $\mathrm{T}$ cells might let the occasional infected cell slip through. In this paper, we argue, through the use of a mathematical model of $\mathrm{T}$ cell and humoral responses to infection, that it is in fact the immediate availability of the neutralizing antibodies, in the secondary response, that renders a vaccine based on a humoral response more effective.

In this paper we put forward the view that the efficacy of the vaccine depends on the reproductive number $\left(R_{0}\right)$ of the virus in the vaccinated host. Only if an infected cell on average produces more than one newly infected cell the infection will take a hold. This notion can be quantified by means of the basic reproductive number, or $R_{0}$ as it is known in epidemiology (Anderson and May, 1991) and we use this to quantify vaccine efficiency. Vaccines based on a humoral response can protect from infection by decreasing the basic reproductive number to less than one. For a $\mathrm{T}$ cell-based protective vaccine the effector $\mathrm{T}$ cells need to be formed from precursor cells. This will result in a delay in the response and, at least for some time after infection, the reproductive number will exceed one. This gives the virus a chance to reproduce and establish itself. It is therefore neither the magnitude of the immune response nor the mode of interaction with the virus but the almost immediate availability of the neutralizing agents that makes a vaccine based on a humoral response effective. $T$ cells lack this property and are therefore at a systematic disadvantage in their potential to generate a protective secondary response. This has recently been confirmed in a primate vaccine model (Davenport et al., 2004).

Not only are $\mathrm{T}$ cell-based vaccines at a systematic disadvantage, but our models revealed they have a further unexpected property. Because $\mathrm{T}$ cell-based vaccines work through the mobilization of effector $T$ cells from precursors, the number of mobilized effector cells depends on the amount of antigen that is present. Therefore, we theoretically predict that if such vaccines are exposed to high antigen concentrations, as would normally be the case in challenge studies, they could perform reasonably well. However, the same vaccines can fail to protect when exposed to much smaller antigen concentrations, as one would expect to occur during naturally occurring infections. Based on this observation we suggest that antigen concentrations should be varied over a range of concentrations in experimental challenges.

Effector $\mathrm{T}$ cells have the potential to reduce the basic reproductive number of the virus, in a similar way to antibodies. Since effector $\mathrm{T}$ cells live shorter than long-lived plasma cells or memory B cells, T cell-based vaccines cannot give the same long-term protection as vaccines based on a $\mathrm{B}$ cell response. To keep the 
level of effector cells sufficiently high, frequent boosting or continuous stimulation is required. Tentative evidence for this is provided by the study of exposed but uninfected Kenian sex workers, who seemed to be protected from the virus, possibly through frequent exposure to the HIV virus which would boost the immune response (Rowland-Jones et al., 1998). It should be noted that a number of these women seroconverted after they reduced their sexual activity, confirming the idea that continuous boosting might be necessary to confer protection (Kaul et al., 2001).

Although there are no experimental data directly supporting the hypothesis that boosting is less required in B cell-based vaccines than in T cell-based ones, this may be partially due to the fact that $T$ cell-based vaccines trials are relatively new and, hence, not much data are available. Another reason is that $\mathrm{T}$ cell-based vaccines may confer protection to other diseases than $B$ cell-based vaccines do, thus making a direct comparison near impossible. Note that recent studies indicate that effective vaccines depend on the stimulation of both humoral and cell mediated immunity. So far all successful vaccines protect via antibodies (Zinkernagel, 2000).

It is conventionally assumed that long-term antibody responses are maintained by continuous differentiation of B cells into short-lived plasma cells. In an experimental vesicular stomatitis virus (VSV)-mouse model it has been shown that approximately 20,000 plasma cells are sufficient to maintain a neutralizing antibody titer for life (Bachmann et al., 1993, 1994) and that as few as $10 \mathrm{~B}$ cells per day differentiating into plasma cells per day would suffice to balance plasma cell death (Funk et al., 1998). In a lymphocytic choriomeningitis virus (LCMV)-mouse model another mechanism for lasting humoral immunity has been described: long-lived, terminally differentiated plasma cells can secrete antibodies for extended periods of time (over 1 year) in the absence of memory B cells (Slifka et al., 1998). This alternative mechanism of maintenance of humoral immunity lends further support to the assumption that effector cells are readily induced, extending it to the cellular level.

Here we argue that without constant stimulation or boosting, $\mathrm{T}$ cell-based vaccines are unlikely to protect from infection for a sustained period of time. Even if means of continuous stimulation can be provided, the level of effector cells needs to be sufficiently high and should exceed levels normally found during chronic infection. This makes the design of a protective $\mathrm{T}$ cellbased vaccine inherently more difficult than a $\mathrm{B}$ cell vaccine. In view of this, vaccines that provide protection by blocking the virus at the point of entry, either through dendritic cells or mucosal immunity, seem much more promising than vaccines based on a $\mathrm{T}$ cell response.

\section{Acknowledgements}

GAF acknowledges support from the Swiss National Science Foundation and from the European Commission through a Marie Curie Fellowship, Contract No. MEIF-CT-2004-501039.

\section{Appendix}

In the appendix we derive the threshold for precursors, $P_{\text {threshold }}$ using a change of variables. Although this is slightly more elaborate, it has the advantage of holding more generally than the result stated in the main body of the paper.

The dynamics at the beginning of an infection are given by Eqs. (6). An activated precursor cell gives rise to $n$ effector cells, therefore the potential of the $\mathrm{T}$ cell population is, in terms of effector cells, given by $E^{\prime}=$ $n P+E$. The fraction of this potential that is available as effector cells is given by $F=E / E^{\prime}$. We will express the dynamics in the new variables $E$ and $F$. These new variables change as

$$
\begin{aligned}
\frac{1}{v+m} \frac{\mathrm{d} E^{\prime}}{\mathrm{d} t}= & \left(\frac{n m}{v+m}-1\right) F E^{\prime}, \\
\frac{1}{v+m} \frac{\mathrm{d} F}{\mathrm{~d} t}= & \frac{b I}{v+m}-\left(\frac{b I}{v+m}+1\right) F \\
& -\left(\frac{n m}{v+m}-1\right) F^{2} .
\end{aligned}
$$

We have put the factor $1 /(v+m)$, which is the average lifetime of effector cells, on the left-hand side to indicate that this represents the natural time-scale of the dynamics of the T cells.

Eq. (8) shows that the total effector potential of the T cell population, $E^{\prime}$, will only increase if $m n /(v+m)>1$. The probability of an effector cell to turn into a memory cell is given by $m /(v+m)$. The expected number of memory cells following proliferation of a single precursor cell is therefore $n m /(v+m)$. The net gain in memory cells is given by $n m /(v+m)-1$ because the precursor cell that proliferated is lost for the precursor population. The $\mathrm{T}$ cell population can only expand if the net number of memory cells produced per activated precursor is positive.

For the sake of argument, we will consider what happens if $v+m$ is large compared to $I$, i.e. the dynamics of the $T$ cells are much faster than the rate of increase of the number of infected cells. If this is the case the lefthand sides of Eqs. $(8,9)$ will be very small. In this case the fraction $F$ equilibrates, and it will do this quickly compared to the dynamics of the infected cells.

To calculate the rate of expansion we need to know the fraction of the $\mathrm{T}$ cell potential that is available as 
effector cells, $F$. This fraction changes over time as it will depend on the number of infected cells. Note, however, that Eq. (9) is independent of $E^{\prime}$. The quasi steady state of $F$ is given by

$\hat{F}=\frac{-1-\frac{b I}{v+m}+\sqrt{\left(1+\frac{b I}{v+m}\right)^{2}+4 \frac{b I}{v+m}\left(\frac{n m}{v+m}-1\right)}}{2\left(\frac{n m}{v+m}-1\right)}$.

If $v+m$ is large relative to the number of infected cells, $I$, the equilibrium is approximately equal to $\hat{F} \approx b I /(v+$ $m$ ) (we found this by expanding $\hat{F}$ in $I /(v+m)$ up to first order). If we substitute this approximation in Eq. (8) we find

$\frac{\mathrm{d} E^{\prime}}{\mathrm{d} t}=b\left(\frac{n m}{v+m}-1\right) I E^{\prime}$.

This equation is similar to Eq. (2) which describes the proliferation of $\mathrm{B}$ cells: the growth rate of both populations is proportional to the amount of antigen times the proliferation constant (a resp. $b(n m /(v+m)-1))$. In the case of $\mathrm{T}$ cells we see that the proliferation constant is proportional to the net number of memory cells produced per activated precursor.

The number of effector cells depends on the number of precursors as $E=n P E /(1-F) \approx P n b I /(v+m)$. Using this we find that the threshold level for the precursors is given by Eq. (7).

\section{References}

Anderson, R.M., May, R.M., 1991. Infectious Diseases of Humans. Oxford University Press, Oxford.

Antia, R., Pilyugin, S.S., Ahmed, R., 1998. Models of immune memory: On the role of cross-reactive stimulation, competition, and homeostasis in maintaining immune memory. Proc. Natl Acad. Sci. USA. 95, 14926-14931.

Antia, R., Bergstrom, C.T., Pilyugin, S.S., Keach, S.M., Ahmed, R., 2003. Models of CD8+ responses: 1. What is the antigenindependent proliferation program. J. Theor. Biol. 221, 585-598.

Bachmann, M.F., Kundig, T.M., Kalberer, C.P., Hengartner, H., Zinkernagel, R.M., 1993. Formalin inactivation of vesicular stomatitis virus impairs T-cell- but not T-help-independent B-cell responses. J. Virol. 67, 3917-3922.

Bachmann, M.F., Kundig, T.M., Kalberer, C.P., Hengartner, H., Zinkernagel, R.M., 1994. How many specific B cells are needed to protect against a virus? J. Immunol. 152, 4235-4241.

Badovinac, V.P., Porter, B.P., Hardy, J.T., 2002. Nat. Immunol. 3, 619-626.

Blattman, J.N., Cheng, L.E., Greenberg, P.D., 2002. CD8 + T cell repsonses: it's all downhill after their prime. Nat. Immunol. 3, 601-602.

Bonhoeffer, S., Rembiszeski, M., Ortiz, G.M., Nixon, D.F., 2000. Risks and benefits of strategic therapy interruptions in HIV-1 infection. AIDS 14, 2313-2322.

Davenport, M.P., Ribeiro, R.M., Perelson, A.S., 2004. Kinetics of virus-specific CD8 $+\mathrm{T}$ cells and the control of human immunodeficiency virus infection. J. Virol. 78, 10096-10103.
De Boer, R.J., Perelson, A.S., 1994. T cell repertoires and competitive exclusion. J. Theor. Biol. 169, 375-390.

Fayaz, A., Simani, S., Nour-Salehi, S., Bahmanyar, M., 1981. Booster effect of human diploid cell antirabies vaccine in previously treated persons. JAMA 20, 2334-2335.

Funk, G.A., Barbour, A.D., Hengartner, H., Kalinke, U., 1998. Mathematical model of a virus-neutralizing immunglobulin response. J. Theor. Biol. 195, 41-52.

Funk, G.A., Fischer, M., Joos, B., Opravil, M., Günthard, H.F., Ledergerber, B., Bonhoeffer, S., 2001. Quantification of in vivo replicative capacity of HIV-1 in different compartments of infected cells. J. AIDS 26, 397-404.

Gruters, R.A., Van Baalen, C.A., Osterhaus, A.D.M.E., 2002. The advantage of early recognition of HIV-infected cells by cytotoxic T-lymphocytes. Vaccine 20, 2011-2015.

Jacob, J., Baltimore, D., 1999. Modelling T-cell memory by genetic marking of memory T cells in vivo. Nature 399, 593-597.

Johnson, R.P., 2002. Mechanisms of protection against simian immunodeficiency virus infection. Vaccine 20, 1985-1987.

Kaech, S.M., Ahmed, R., 2001. Memory CD8 + T cell differentiation: initial antigen encounter triggers a developmental program in naive cells. Nat. Immunol. 2, 415-422.

Kaul, R., Rowland-Jones, S.L., Kimani, J., Dong, T., Yang, H.B., Kiama, P., Rostron, T., Njagi, E., Bwayo, J.J., MacDonald, K.S., McMichael, A.J., Plummer, F.A., 2001. Late seroconversion in HIV-resistant Nairobi prostitutes despite pre-existing HIV-specific CD8(+) responses. J. Clin. Invest. 107, 341-349.

Korthals Altes, H., Price, D.A., Jansen, V.A.A., 2002. Effector cytotoxic $\mathrm{T}$ lymphocyte numbers induced by vaccination should exceed levels in chronic infection for protection from HIV. Vaccine 20, 3-6.

Little, S.J., McLean, A.R., Spina, C.A., Richman, D.D., Havlir, D.V., 1999. Viral dynamics of acute HIV-1 infection. J. Exp. Med. 190, 841-850.

Maecker, T.M., Maino, V.C., 2003. T cell immunity to HIV: defining parameters of protection. Curr. HIV Res. 1, 249-259.

Maruyama, M., Lam, K.P., Rajewsky, K., 2000. Memory B-cell persistence is independent of persisting immunizing antigen. Nature 407, 636-642.

McLean, A.R., 1994. Modelling T-cell memory. J. Theor. Biol. 170, 63-74.

McMichael, A., Mwau, M., Hanke, T., 2002. HIV T cell vaccines, the importance of clades. Vaccine 20, 1918-1921.

Nowak, M.A., May, R.M., 2000. Virus Dynamics. Oxford University Press, Oxford.

Ogg, G.S., Jin, X., Bonhoeffer, S., Moss, P., Nowak, M.A., Monard, S., Segal, J.P., Cao, Y., Rowland-Jones, S.L., Hurley, A., Markowitz, M., Ho, D.D., McMichael, A.J., Nixon, D.F., 1999. Decay kinetics of human immunodeficiency virus-specific effector cytotoxic $\mathrm{T}$ lymphocytes after combination antiretroviral therapy. J. Virol. 73, 797-800.

Perelson, A.S., 2000. Modelling viral and immune system dynamics. Nat. Rev. Immun. 2, 28-36.

Robbins, J.B., Schneerson, R., Szu, S.C., 1995. Perspective: hypothesis: serum IgG antibody is sufficient to confer protection against infectious diseases by inactivating the inoculum. J. Infect. Dis. 171, $1387-1398$

Rowland-Jones, S.L., Dong, T., Fowke, K.R., Kimani, J., Krausa, P., Newell, H., et al., 1998. Cytotoxic T cell responses to multiple conserved HIV epitopes in HIV-resistant prostitutes in Nairobi. J. Clin. Invest. 102, 1758-1765.

Seder, R.A., Ahmed, R., 2003. Similarities and differences in CD4+ and CD $8+$ effector and memory $\mathrm{T}$ cell generation. Nat. Immunol. $4,835-842$.

Slifka, M.K., Antia, R., Whitmire, J.K., Ahmed, R., 1998. Humoral immunity due to long-lived plasma cells. Immunity $8,363-372$. 
Takahashi, Y., Ohta, H., Takemori, T., 2001. Fas is required for clonal selection in germinal centers and the subsequent establishment of the memory B cell repertoire. Immunity 14, 181-192.

Utzny, C., Burroughs, N.J., 2001. Stability of a diverse immunological memory is determined by $\mathrm{T}$ cell population dynamics. Bull. Math. Biol. 63, 685-713.

Van Stipdonk, M.J.B., Lemmens, E.E., Schoenberger, S.P., 2001. Naive CTLs require a single brief period of antigenic stimulation for clonal expansion and differentiation. Nat. immunol. 2, 423-429.
Welsch, J.A., Granoff, D., 2004. Naturally acquired passive protective activity against Neisseria meningitidis Group C in the absence of serum bacterial activity. Infect. Immun. 72, 5903-5909.

Wodarz, D., Jansen, V.A.A., 2001. The role of T Cell help for antiviral CTL responses. J. Theor. Biol. 211, 419-432.

Zinkernagel, R.M., 2000. What is missing in immunology to understand immunity? Nat. Immunol. 1, 181-185.

Zinkernagel, R.M., 2002. Immunity, immunopathology and vaccines against HIV? Vaccine 20, 1913-1917. 